\title{
Excitonic Effects in X-Ray Transitions in Metals*
}

\author{
G. D. Mahan ${ }^{1}$ \\ Institute of Theoretical Science and Department of Physics, University of Oregon, Eugene, Oregon $94703^{2}$ \\ and
}

General Electric Research and Development Center, Schenectady, New York 12301

(October 10, 1969)

\begin{abstract}
In the study of soft x-ray transitions in solids, there has always been some hope that the results provide a direct measure of the density of states. This assumes that (a) matrix element variations over the band and (b) final state interactions are small. Both of these assumptions are now known to be incorrect. To illustrate the possible strength of these effects, two approximate calculations are presented: the one electron oscillator strength of a simple bec metal as a function of energy; and the strength of the Nozieres-DeDominicis singularity at threshold, with phase shifts estimated from an assumed Yukawa interaction between conduction electrons and core hole.
\end{abstract}

Key words: Density of states; exciton; many body effects; phase shifts; soft x-ray; transition probability.

\section{Introduction}

The absorption of a photon can cause an electron to change its state. The traditional viewpoint of this transition assumes that if one knew the electrons initial $\psi_{i}$ and final $\psi_{f}$ state, then the oscillator strength was simply proportional to the square of the momentum matrix element.

$$
p_{i f}=\int d^{3} r \psi_{f}^{*} p \psi_{i}
$$

This simple viewpoint is now known to be incorrect. The proper picture is that the optical transition creates an electron and hole, and these two excitations interact with each other, and each separately with their environment. The coulomb scattering between the electron and hole is called the exciton effect, named after the first known example of Frankel excitons in solids. The electron and hole can also emit phonons, suffer electron-electron collisions, etc. The sum of all of these processes are called final-state interactions. The rate

*An invited paper presented at the 3d Materials Research Symposium, Electronic Density of States, November 3-6, 1969, Gaithersburg, Md.

Alfred P. Sloan-Research Fellow.

${ }^{2}$ Research Supported by National Science Foundation Grant. of optical absorption is affected by these subsequent interactions of the electron and hole.

Upon reviewing our present understanding of the optical properties of solids, one finds that some solids are well understood while others are not. We classify as understood the semiconductors Si and Ge, [1] and simple metals like aluminum [2,3]. It is noteworthy that in these materials the final-state interactions are small: they are small in semiconductors because the large dielectric constant suppresses the exciton effect. Ehrenreich and his co-workers have shown that many body effects are small in aluminum. In these solids the simple one-electron picture implied in (1.1) seems to work quite well.

We classify wide band gap insulators [4], and metals such as copper [5], as solids whose optical properties are not well understood. There is not yet good agreement between theory and experiment in these materials. The dominant optical transitions in these materials create hole states which are heavy, and which are subject to significant final state interactions [3]. We speculate that final state interactions are important in these materials, and explain the difference between theory and experiments.

In the study of x-ray transition in solids, there has always been some hope that the results provide a direct 
measure of the density of states [6]. This presumes that (a) matrix element variations over the band are small, and (b) final state interactions are small. Both of these assumptions are now known to be incorrect $[7,8,9]$.

The importance of exciton effects in $\mathrm{x}$-ray trarisitions was reported in an earlier reference [10]. The effects are large if the hole is highly localized, and if the conduction band of the electron is isotropic. Because the coulomb scattering between the electron and hole occurs in an electron gas, the electron can only scatter into states not already occupied. This Fermi-Dirac exclusion, as well as the exchange interaction among the electrons, causes the problem to resemble the Kondo effect more than the Wannier-exciton case. The hole also has a large interaction with the entire electron gas - this leads to a renormalization effect which inhibits the x-ray transition near threshold [11]. Nozieres and DeDominicis [12] have shown that the exciton and renormalization effects combine to give a threshold behavior for $\epsilon_{2}(\omega)$

$$
\epsilon_{22}(\omega) \sim \frac{1}{\omega^{2}}\left(\frac{\xi_{0}}{\omega-\omega_{T}}\right)^{\alpha_{l}} \theta\left(\omega-\omega_{T}\right)
$$

where $\omega_{T}$ is the absorption threshold frequency, $\xi_{0} \sim$ $E_{F}$ is a characteristic band energy, and $\alpha_{l}$ is a function of the phase shifts $\delta_{l}$ of electrons at the Fermi energy scattering from the static potential of the hole.

$$
\alpha_{l}=\frac{2 \delta_{l}}{\pi}-2 \sum_{l=0}^{\infty}(2 l+1)\left(\frac{\delta_{l}}{\pi}\right)^{2}
$$

The term $2 \delta_{l} / \pi$ is the exciton part which tends to make the threshold singular, while the second term in (1.3) arises from renormalization. The angular momentum $l$ is that of the conduction electron [13]. If the x-ray hole has s-symmetry, the conduction electron must have psymmetry $(l=1)$. If the hole has p-symmetry, the conduction electron can have either s- or d-symmetry; in this case (1.2) has a separate term for each symmetry type.

The $x$-ray spectra can only be unravelled with a knowledge of the phase shifts $\delta_{l}$-these are the phase shifts for simple electron-hole scattering. These have been calculated for a free electron gas by Ausman and Glick [13]. They find $\alpha_{0}>0$, and $\alpha_{l}<0$ for $l \geqslant 1$ so that singularities only occur for the $l=0$ case (p-state hole). These results qualitatively agree with the experimental results.

We have independently calculated the phase shifts, and we describe our result in section II. We have concluded that these phase shifts are qualitatively correct but for the wrong reasons. The wrong potential is used in the calculation, but it does not seem to make much difference in this case.

\section{Phase Shifts}

The present calculations have been performed by assuming that the screened electron-hole interaction has the Yukawa form

$$
V(r)=-\frac{e^{2}}{r} \exp \left(-k_{s} r\right)
$$

where the Fermi-Thomas screening length is

$$
k_{s}^{2}=6 \pi e^{2} n_{0} / E_{F}
$$

for an electron gas of density $n_{0}$ and Fermi energy $E_{f}$. This is only a crude approximation to the actual potential the electron feels when scattering from the hole. For example, studies of a point charge impurity in an electron gas show that its potential differs from a $\mathrm{Yu}$ kawa at long range where Friedel oscillations occur, and at short range where its potential is less steep [14]. In addition, because the hole is part of an atom, there are term due to the exchange and orthogonality with the atomic wave functions $[7,8]$. In a pseudo-potential formalism these latter effects contribute a short-range repulsive term to the interaction. In spite of these shortcomings of the Yukawa potential, we believe that it predicts phase shifts which are qualitatively correct. The reasons for this will be presented below.

The phase shifts are defined in terms of the eigenvalues $E_{k}$ and eigen functions $\psi_{k}(r)$ of the electron in the region of the potential.

$$
\left(-\frac{\nabla^{2}}{2 m}+V(r)-E_{k}\right) \psi_{k}(r)=0
$$

The wave is decomposed into spherical harmonics

$$
\psi_{k}(r)=\sum_{l=0}^{\infty}(2 l+1) i^{l} P_{l}(\hat{k} \cdot \hat{r}) \phi_{l}(k, r)
$$

In solving for the eigen functions in (2.1), one has the choice of specifying the boundary conditions for states which are plane wave-like outside of the potential region. By choosing standing wave conditions, one is solving for a reaction matrix $K_{l}\left(k, k^{\prime}\right)$ and the phase shifts are defined in terms of the diagonal $k=k^{\prime}$ component [15].

$$
\begin{aligned}
\tan \delta_{l}(k) & =-2 m k K_{l}(k, k) \\
& =-2 m k \int_{0}^{\infty} r^{2} d r j_{l}(k r) V(r) \phi_{l}(k, r)
\end{aligned}
$$


Similarly, if one chooses outgoing wave conditions, then one is solving for a T-matrix $t_{l}\left(k, k^{\prime}\right)$ whose diagonal components give the phase shift [15].

$$
\begin{aligned}
\sin \delta_{l} e^{i \delta_{l}}= & -2 m k t_{l}(k, k) \\
& =-2 m k \int_{0}^{\infty} r^{2} d r j_{l}(k r) V(r) \phi_{l}(k, r)
\end{aligned}
$$

We have chosen to use the reaction-matrix formalism (2.3), mostly because it is a real function and this simplifies computation.

The phase shifts $\delta_{l}(k)$ go to zero as $k \rightarrow \infty$. Let us now examine their behavior as $k \rightarrow 0$. From (2.3) we have $j_{l}(k r) \sim(k r)^{l}$, so

$$
\tan \delta_{l}(k) \sim k^{l+1} \int_{0}^{\infty} r^{l+2} d r V(r) \phi_{l}(0, r)
$$

From Levinson's Theorem we know that

$$
\delta_{l}(0)=\pi M_{l}
$$

where $M_{l}$ is the number of bound states of (2.1) with angular momentum $l$. For example, if $M_{l}=0$ we get $\delta_{l} \sim k^{l+1}$. Whereas if $M_{l}=1$ we get $\delta_{l}=\pi+c k^{l+1}$ (where $c$ is some constant) for small $k$. A typical case is shown in figure 1 , where $M_{0}=1$, and $M_{l}=0$ for $l \geqslant 1$. So the s-wave phase shift comes into $\pi$ with a linear slope, while all other $\delta_{l} \sim k^{l+1}$ at small $k$.

So in calculating phase shifts, the first thing to decide is the number of bound states $M_{l}$. This is determined by examining the radial part of Schrödinger's equation (2.1) which we put into dimensionless form

$$
\begin{gathered}
{\left[-\frac{\partial^{2}}{\partial \rho^{2}}+\frac{l(l+1)}{\rho^{2}}-\lambda \frac{e^{-\rho}}{p}-\mathscr{E}\right] r \phi_{l}=0} \\
\rho=k_{s} r \\
\lambda=\frac{2 m e^{2}}{\hbar^{2} k_{s}}=\left(\frac{2 \pi}{3}\right)^{1 / 3} \sqrt{r_{s}} \\
\mathscr{E}=2 m E / \hbar^{2} k_{s}^{2}
\end{gathered}
$$

The parameter $\lambda$ determines the strength of the potential. Shey and Schwartz [16] have computed the number of bound states which exist for each value of $\lambda$ and $l$. For s-waves $(l=0)$, they find no bound states exist for $\lambda<1.68$, one bound state for $1.68<\lambda<6.45$, two bound states for $6.45<\lambda<14.3$, et $c$. For p-waves $(l=1)$ bound states only exist for $\lambda>9.08$, and $d$-wave bound states exist for $\lambda>21.8$.
In (2.7) we have written $\lambda$ in terms of the density parameter $r_{s}$ for an electron gas. Since metallic densities vary between $2<r_{s}<6$, then the range of $\lambda$ values in metals is $1.8<\lambda<3.1$. For an electron gas of metallic density, there is one bound state for s-states, and no bound states for any other value of angular momentum $l$.

These are the predictions of the Yukawa potential. We must decide whether these are reasonable conclusions for the problem of interest. For an actual point charge in an electron gas, e.g., a proton, there is probably a bound state. For an impurity in a host metal, e.g., $\mathrm{Al}$ atoms in $\mathrm{Mg}$, there is probably not a real bound state. This is because the atomic core of the impurity cuts off the attractive potential in the region where it is the strongest; for example, see Ashcroft's remarks about the cancellation influence of the atomic cores [8].

In the x-ray problem there is certainly an atomic core. Yet there is also certainly a bound state in the potential. This bound state is the $\mathrm{x}$-ray level itself. That is, if an electron scattering from the potential of the hole did not think a bound state existed in the potential, then it would have no inclination to recombine with the hole in the final emission process. Since the emission can occur, a bound state does exist which must be reflected on the phase shifts.

The cancellation of the potential in atomic core is caused by the necessity of the conduction electron wave function to be orthogonal to all of the core states. In the $\mathrm{x}$-ray problem, one core electron is absent so that the cancellation requirements are less stringent.

The foregoing discussion shows that any phase shifts calculated from a simple Yukawa potential are only going to be qualitatively correct. Yet there is some interest in what this simple model predicts. Our method of calculation proceeds by solving directly the scattering equation for the reaction matrix.

$$
\begin{aligned}
& K_{l}\left(k_{1}, k_{2}\right)=V_{l}\left(k_{1}, k_{2}\right) \\
& \quad+\frac{4 m}{\pi} \int_{0}^{\infty} k_{3}^{2} d k_{3} \frac{V_{l}\left(k_{1}, k_{3}\right) K_{l}\left(k_{3}, k_{2}\right)}{k_{2}^{2}-k_{3}^{2}}
\end{aligned}
$$

where the $l^{\text {th }}$ component of the potential $V_{l}$ is obtained from the Fourier transform $V\left(\mathbf{k}_{1}-\mathbf{k}_{2}\right)$ of $V(r)$

$$
\begin{aligned}
V_{l}\left(k_{1}, k_{2}\right) & =\frac{1}{2} \int_{0}^{\pi} d \theta \sin \theta P_{l}(\cos \theta) V\left(\mathbf{k}_{1}-\mathbf{k}_{2}\right) \\
& =\frac{e^{2}}{2 k_{1} k_{2}} Q_{l}\left(\left(k_{1}^{2}+k_{2}^{2}+k_{s}^{2}\right) / 2 k_{1} k_{2}\right)
\end{aligned}
$$


when $\cos \theta$ is the angle between $\mathbf{k}_{1}$ and $\mathbf{k}_{2}$, and $Q_{l}$ is a Legendre function. We will abbreviate the argument of the Legendre function to write it as $Q_{l}\left(k_{1}, k_{2}\right)$. Noyes' method is used to evaluate (2.8). We find

$$
\begin{gathered}
K_{l}(k, k)=V_{l}(k, k) /\left(1-\Lambda_{l}(k)\right) \\
\Lambda_{l}(k)=\frac{-4 m}{\pi V_{l}(k, k)} \int_{0}^{\infty} \frac{p^{2} d p}{p^{2}-k^{2}} V_{l}(k, p) f_{l}(k, p) \\
f_{l}(k, p)=V_{l}(k, p) \\
+\frac{4 m}{\pi V_{l}(k, k)} \int_{0}^{\infty} \frac{p^{\prime 2} d p^{\prime} f_{l}\left(k, p^{\prime}\right)}{p^{\prime 2}-k^{2}}\left[V_{l}(p, k) V_{l}\left(k, p^{\prime}\right)\right. \\
\left.-V_{l}\left(p, p^{\prime}\right) V_{l}(k, k)\right]
\end{gathered}
$$

One obtains $f_{l}(k, p)$ from (2.12), perhaps by iterating this equation. This is used in (2.11) to obtain $\Lambda_{l}(k)$, and thus one has the reaction matrix in (2.10). This is an exact result if $f_{l}(k, p)$ is found exactly. The Born Approximation result is obtained by setting $K_{l}(k, k)=$ $V_{l}(k, k)$.

Note that it is natural to write $\Lambda_{l}(k)$ as a power series in the interaction strength $\lambda=2 / k_{s} a_{B}$ in (2.7)

$$
\Lambda_{l}(k)=\sum_{m=1}^{\infty} \lambda^{m} g_{l}^{(m)}\left(k / k_{s}\right)
$$

Each successive power of $\lambda$ corresponds to another iteration of the equation (2.12) which determines $f_{l}(k, p)$. For example, the first term is

$$
g_{l}^{(1)}\left(k_{i}\right)=\frac{k_{s}}{\pi Q_{l}(k, k)} \int_{0}^{\infty} \frac{d p}{p^{2}-k^{2}} Q_{l}(k, p)^{2}
$$

For $l=0$ we can evaluate the integral and express the integral as a summation.

$$
\begin{array}{r}
g_{0}^{(1)}(k)=\frac{k_{s}}{2 k Q_{0}(k, k)} \sum_{l=1}^{\infty} \frac{(-)^{l}}{l^{2}}\left[2 \rho^{l} \sin (\varphi l)\right. \\
-\sin (2 l \varphi)] \\
\rho=\left[1+4 k^{2} / k_{s}^{2}\right]^{-1 / 2} \\
\varphi=\tan ^{-1}\left(2 k / k_{s}\right) \\
Q_{0}(k, k)=\frac{1}{2} \ln \left(1+4 k^{2} / k_{s}^{2}\right)
\end{array}
$$

This form is convenient for numerical computation. In figure 1 is shown the s-wave phase shift calculated by approximating $\Lambda_{0}$ by the first term in (2.13)

$$
\tan \delta_{0}(k)=\frac{Q_{0}(k, k)}{k a_{B}\left[1-\lambda g_{0}^{(1)}(k)\right]}
$$

Also shown in figure 1 are the phase shifts $\delta_{1}$ and $\delta_{2}$. We also calculated the first correction term $g_{1}^{(1)}(k)$ to

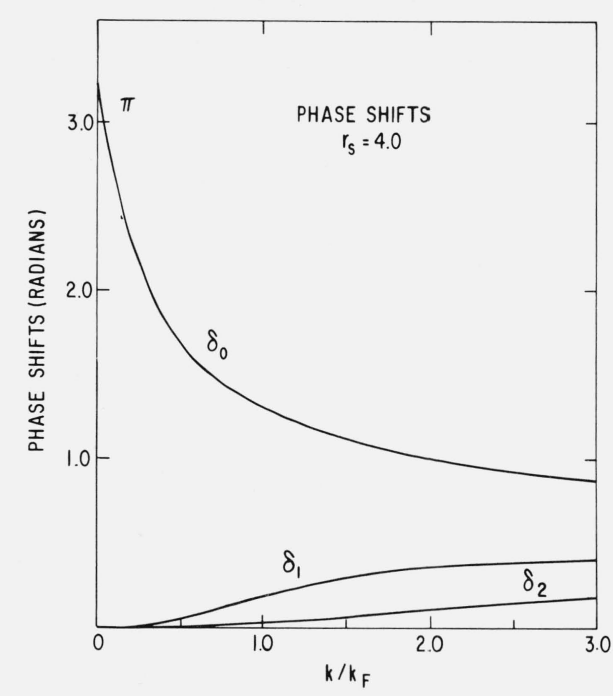

FigurE 1. The phase shifts $\delta_{l}$ calculated by a Yukawa potential. This potential represents a coulomb interaction of unit charge, with Fermi-Thomas screening. The phase shift $\delta_{2}$ is calculated in the Born Approximation, while $\delta_{0}$ and $\delta_{1}$ have corrections for multiple scattering.

the p-wave phase shift, and this correction has been added into the calculation of the curve labeled $\delta_{1}$. This only changes the Born Approximation result by 10 percent. The $l=2$ phase shift is the Born Approximation result. The multiple scattering terms are small except for the s-wave.

In the x-ray transition, the singular effects occur at the Fermi surface, so we are interested in the phase shifts evaluated at $k_{f}$. Figure 2 shows the critical ex-

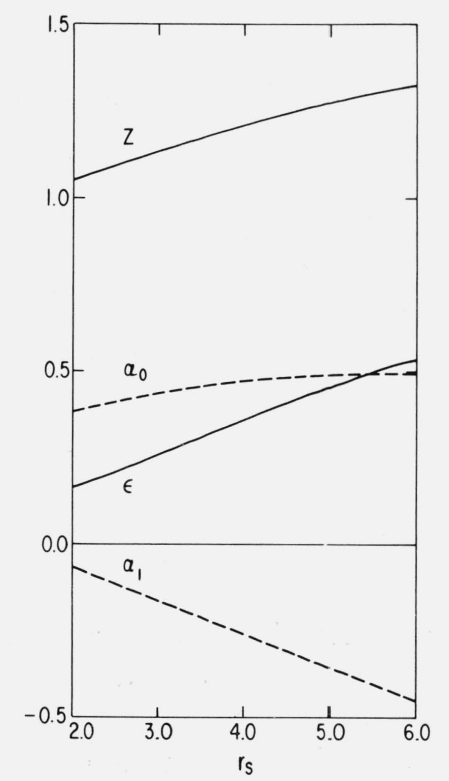

FIGURE 2. The variation with electron density $r_{s}$ of the exponents $\alpha_{0}$ and $\alpha_{1}$. These depend upon the phase shifts evaluated at the Fermi surface. Also shown is the renormalization parameter $\epsilon$ and the $F$ riedel sum rate result $Z$. The value of $Z$ is rigorously unity, and our deviations from that value indicate the errors in the calculation. 
ponents $\alpha_{0}$ and $\alpha_{1}$, in (1.3) plotted versus electron density $r_{s}$. Values of $\alpha_{l}$ for $l \geqslant 2$ are essentially equal to $-\epsilon$. Also shown are the values of the Friedel sum $Z$ and the Anderson exponent $\epsilon$, where

$$
\begin{aligned}
& Z=2 \sum_{l=0}^{\infty}(2 l+1)\left(\delta_{l} / \pi\right) \\
& \boldsymbol{\epsilon}=2 \sum_{l=0}^{\infty}(2 l+1)\left(\delta_{l} / \pi\right)^{2}
\end{aligned}
$$

The Friedel sum rule should rigorously be given by $z=1$. Our deviation $\sim 20$ percent from the exact result of $z=1$ provides an estimate of the error in the calculation.

Another estimate of the accuracy and convergence of the calculational method is obtained by seeing how well it estimates the position of bound states. As $k \rightarrow 0$ we get

$$
\lim _{k \rightarrow 0} \delta_{0}(k)=\tan ^{-1}\left(\frac{2 k / k s}{1-\Lambda_{0}(0)}\right)
$$

A bound state is predicted if $\Lambda_{0}>1$. We find that

$$
\begin{aligned}
& g_{0}^{(1)}(0)=1 / 2 \\
& g_{0}^{(2)}(0)=\ln (4 / 3)-1 / 4
\end{aligned}
$$

If we just use the first term in the expansion (2.13) so $\Lambda_{0}(0)=\lambda / 2$ then the criteria $\Lambda_{0}(0)>1$ means $\lambda>2$. This is an error of 16 percent from the actual criteria $\lambda>1.68$. But using two terms gives $\lambda / 2+\lambda^{2}(\ln$ $4 / 3-1 / 4)>1$ or $\lambda>1.765$. So a considerable improvement in accuracy is obtained by including the second term in (2.13). So we conclude that the series (2.13) converges rapidly for the range of $\lambda$ values of interest.

\section{Matrix Elements}

The change of the matrix element with energy has been discussed before and is known to be a large effect $[7,8,9]$. We have calculated the change in matrix element near the first critical point in a bcc solid for an $\mathrm{x}$ ray transition from a $1 s$ core level. We used a simple two band model in each 1/12 of the Brillouin Zone [17], so that the energies and eigenstates are

$$
\begin{gathered}
E_{k}^{ \pm}=\frac{1}{2}\left(\mathscr{E}_{k}+\mathscr{E}_{\mathbf{k}-\mathbf{G}}\right) \pm\left[\frac{1}{4}\left(\mathscr{E}_{k}-\mathscr{E}_{\mathbf{k}-\mathbf{G}}\right)^{2}+V_{i}^{2}\right]^{1 / 2} \\
\psi_{\bar{k}}^{ \pm}(r)=N_{k}\left\{\left|\mathscr{E}_{k}-E_{k}^{\mp}\right|^{1 / 2} e^{i \mathbf{k} \cdot \mathbf{r}} \pm \frac{V_{G}}{\left|V_{G}\right|}\left|\mathscr{E}_{k}-E_{k}^{ \pm}\right| 1 / 2 e^{i r \cdot(\mathbf{k}-\mathbf{G})}\right\} \\
N_{k}=\frac{1}{2}\left[\frac{1}{4}\left(\mathscr{E}_{k}-\mathscr{E}_{\mathbf{k}-\mathbf{G}}\right)^{2}+V_{G}^{2}\right]^{-1 / 2}
\end{gathered}
$$

The matrix elements were calculated assuming that the core was a delta function, and no attempt was made to orthogonalize the conduction states to the core. Thus, after averaging over polarizations, the matrix elements are

$$
\begin{aligned}
\left\langle p_{k}^{2}\right\rangle \pm=\frac{1}{3} N_{k}^{2}\left\{k^{2}\left|\mathscr{E}_{k}-E_{k}^{\mp}\right|+(\mathbf{k}-\mathbf{G})^{2} \mid \mathscr{E}_{k}\right. & \\
& \left.-E_{\frac{1}{+}}^{+} \mid \pm 2 \mathbf{k} \cdot(\mathbf{k}-\mathbf{G}) V_{i}\right\}
\end{aligned}
$$

In figure 3 we show the density of states $\rho(E)$, and also the absorption strength

$$
A(E)=\int \frac{d^{3} k}{(2 \pi)^{3}}\left\{\left\langle p_{k}^{2}\right\rangle+\delta\left(E-E_{k}^{+}\right)+\left\langle p_{k}^{2}\right\rangle-\delta\left(E-E_{k}^{-}\right)\right\}
$$

In this figure, energy has been normalized to $E_{0}=$ $\hbar^{2} G^{2} / 8 m$ so that $\mathscr{E}=E / E_{0}$ and $\nu=V_{G} / E_{0}$. The choice $\nu$ $=0.2$ is close to Ham's value for $\operatorname{Li}(\nu=0.23)$. Indeed, the present calculation was done with $\mathrm{Li}$ in mind, since the lack of core orthogonalization should not matter here.

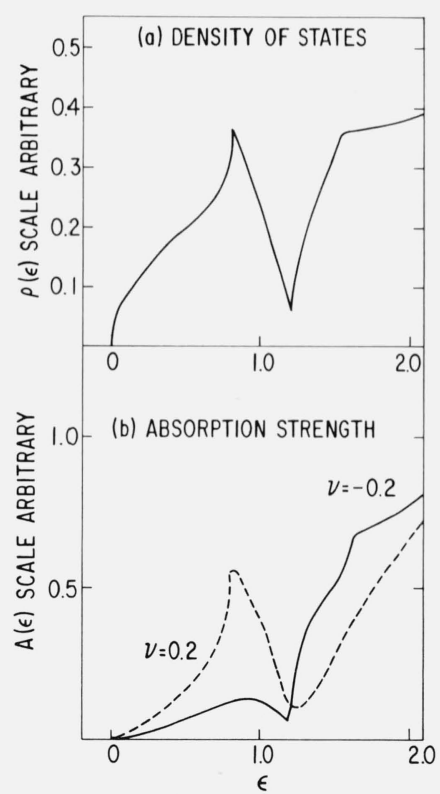

Figure 3. The density of states $\rho(\epsilon)$ and absorption strength $A(\epsilon)$ for a bcc solid such as Li. The energy scale is listed in units $E_{0}=\hbar^{2} G^{2}{ }_{110} / 8 m$, and $\nu=V_{110} / E_{0}$. The absorption strength $A(\epsilon)$ is defined as the integral of the $x$-ray matrix element $\left\langle p^{2}\right\rangle$ averaged over the energy band.

The curve for $\nu=0.2$ in figure 3(b) has the expected shape for $\mathrm{Li}$ [18]. Because the wave function is p-like at the lower critical point $\mathscr{E}=0.8$, the transition is allowed and the absorption strength has the same shape as the density of states in figure $3 \mathrm{a}$. The density of states structure at $\mathscr{E}=1.2$ is washed out in $\mathrm{A}(\mathscr{E})$ because at the critical point the transition is s-like and largely 
forbidden. The curve for $\nu=-0.2$ has the same density of states, but now the upper critical point is p-like and $\mathbf{A}(\mathscr{E})$ has the same structure as $\rho(\mathscr{E})$ near 1.2. Neither the curve $\nu=0.2$ nor $\nu=-0.2$ has any striking resemblance to the density of states [18].

\section{Discussion}

Exciton effects should influence nearly all parts of the absorption spectra. The singular behavior at the absorption or emission edge is just one prominent feature. Another result of final state interactions is that oscillator strength is moved from one frequency range to another. Often these shifts are small and can be neglected. Yet in most cases the true magnitude of these effects are unknown, because the relevant theoretical calculations are too complicated to do realistically.

The calculation of exciton effects is a formidable task which has not been performed properly. Of course Wannier excitons have been studied in detail. Some model calculations exist near critical point edges - the hyperbolic excitons $[19,20,21]$. But the main optical absorption strength comes from states throughout the Brillouin Zone [5], and all of these states are included in the final state interactions.

So before x-ray absorption and emission measurements can be used to provide information on the density of states, two sizable corrections need to be made. One of these is the exciton effect, and the other is the change in the matrix element with energy.

\section{Acknowledgments}

I wish to thank N. Ashcroft, L. Parrott, and M. Stoneham for informative discussions.

\section{References}

[1] Dresselhaus, G., and Dresselhaus, M. S., Phys. Rev. 160, 649 (1967).

[2] Ehrenreich, H., and Beeferman, L., (private communcation).

[3] Spicer, W. E., Phys. Rev. 154, 385 (1967).

[4] Fong, C. Y., Saslow, W., and Cohen, M. L., Phys. Rev. 168, 992 (1968); Park, K., and Stafford, R. G., Phys. Rev. Letters 22, 1426 (1969).

[5] Dresselhaus, G., Sol. State Comm. 7,419 (1969); Mueller, F. M., and Phillips, J. C., Phys. Rev. 157, 600 (1967).

[6] Tombouliam, D. H., Handbuch der Physik, Vol. 30, (SpringerVerlag, Berlin, 1957), 246-304.

[7] Harrison, W. A., Soft X-Ray Band Spectra, edited by D. J. Fabin (Academic Press, New York, 1968), p. 227.

[8] Ashcroft, N. W., ibid, p. 249.

[9] Rooke, G. A., ibid, p. 3.

[10] Mahan, G. D., Phys. Rev. 163,612 (1967).

[11] Anderson, P. W., Phys. Rev. Letters 18, 1049 (1967).

[12] Nozieres, P., and DeDominicis, C. T., Phys. Rev. 178, 1097 (1969).

[13] Ausman, G. A., and Glick, A., Phys. Rev.

[14] Langer, J. S., and Vosko, S. H., J. Phys. Chem. Solids 12, 196 (1960).

[15] Goldberger, M. L., and Watson, K. M., Collision Theory (J. Wiley and Sons, New York, 1964), p. 232.

[16] Schey, H. M., and Schwartz, J. L., Phys. Rev. 139, Bi428 (1965).

[17] Foo, E-Ni, and Hopfield, J. J., Phys. Rev. 173, 635 (1968).

[18] Shaw, R. W., Smith, K., and Smith, N. V., Phys. Rev. 178, 985 (1969).

[19] Duke, C. B., and Segall, B., Phys. Rev. Letters 17, 19 (1966).

[20] Hermanson, J., Phys. Rev. Letters 18, 170 (1967).

[21] Kane, E. O., Phys. Rev. 180, 852 (1969).

(Paper 74A2-598) 